OPEN ACCESS

Edited by: Mikhail Lebedev, Duke University, USA

Reviewed by: Jaeseung Jeong, Korea Advanced Institute of Science and Technology (KAIST), South Korea Evgueni Blagovechtchenski, National Research University - Higher School of Economics, Russia

${ }^{*}$ Correspondence: Thomas A. Deuel tasdeue/@gmail.com

Received: 05 August 2016 Accepted: 11 April 2017

Published: 26 April 2017

Citation:

Deuel TA, Pampin J, Sundstrom J and Darvas F (2017)

The Encephalophone: A Novel Musical Biofeedback Device using Conscious Control of Electroencephalogram (EEG).

Front. Hum. Neurosci. 11:213. doi: 10.3389/fnhum.2017.00213

\section{The Encephalophone: A Novel Musical Biofeedback Device using Conscious Control of Electroencephalogram (EEG)}

\author{
Thomas A. Deuel ${ }^{1,2 *}$, Juan Pampin ${ }^{2,3}$, Jacob Sundstrom ${ }^{3}$ and Felix Darvas ${ }^{4}$ \\ ${ }^{1}$ Department of Neurology, Swedish Neuroscience Institute, Seattle, WA, USA, ${ }^{2}$ Center for Digital Arts and Experimental \\ Media (DXARTS), University of Washington, Seattle, WA, USA, ${ }^{3}$ School of Music, University of Washington, Seattle, WA, \\ USA, ${ }^{4}$ Department of Neurosurgery, University of Washington, Seattle, WA, USA
}

A novel musical instrument and biofeedback device was created using electroencephalogram (EEG) posterior dominant rhythm (PDR) or mu rhythm to control a synthesized piano, which we call the Encephalophone. Alpha-frequency (8-12 Hz) signal power from PDR in the visual cortex or from mu rhythm in the motor cortex was used to create a power scale which was then converted into a musical scale, which could be manipulated by the individual in real time. Subjects could then generate different notes of the scale by activation (event-related synchronization) or de-activation (event-related desynchronization) of the PDR or mu rhythms in visual or motor cortex, respectively. Fifteen novice normal subjects were tested in their ability to hit target notes presented within a 5-min trial period. All 15 subjects were able to perform more accurately (average of 27.4 hits, $67.1 \%$ accuracy for visual cortex/PDR signaling; average of 20.6 hits, $57.1 \%$ accuracy for mu signaling) than a random note generation (19.03\% accuracy). Moreover, PDR control was significantly more accurate than mu control. This shows that novice healthy individuals can control music with better accuracy than random, with no prior training on the device, and that PDR control is more accurate than mu control for these novices. Individuals with more years of musical training showed a moderate positive correlation with more PDR accuracy, but not mu accuracy. The Encephalophone may have potential applications both as a novel musical instrument without requiring movement, as well as a potential therapeutic biofeedback device for patients suffering from motor deficits (e.g., amyotrophic lateral sclerosis (ALS), brainstem stroke, traumatic amputation).

Keywords: biofeedback, electroencephalogram, music, brain-computer interface, rehabilitation

\section{INTRODUCTION}

Since early in the history of the use of electroencephalogram (EEG) for measurement of electrical patterns of the human brain, efforts have been made to transform EEG electrical activity into sound. These efforts not only created diagnostic alternatives to purely visual feedback, but also opened up new possibilities for artistic expression, and created possibilities for therapeutic biofeedback.

The earliest example of converting EEG signal to sound appears in the literature shortly after the invention of the EEG. Adrian and Matthews (1934), replicating the earliest EEG descriptions of the posterior dominant rhythm (PDR; "the Berger rhythm") by Berger (1929), monitored their own EEG with sound (Adrian and Matthews, 1934). 
Conversion of EEG signals to not just sound, but musical modalities, followed later: in 1965, the composer and experimental musician Lucier (1965) created a performance involving control of percussion instruments via strength of EEG PDR, with the encouragement and participation of composer John Cage. However, they experienced some difficulty in achieving good control, and to overcome this employed a second performer manually adjusting the gain from the EEG output (Rosenboom, 1975).

Following in Lucier's pathway 5 years later, David Rosenboom in 1970 created a performance piece called "Ecology of the Skin" for Automation House in New York, NY, USA. This involved using EEG signal from 10 participants processed through individualized electronic circuits to generate visual and auditory performance (Rosenboom, 1975). More recently, Brouse et al. (2006), created EEG waveform spectral analysis in multiple frequency bands to passively control sound and music, in a project for the eNTERFACE summer workshop.

Eduardo Miranda at the Interdisciplinary Centre for Computer Music Research (ICCMR) at Plymouth University, UK was part of that summer workshop project, and has gone on to contribute significantly in this area of generating music from EEG signal. In 2008, he used the changing patterns of alpha and beta frequency rhythms in EEG to act as a switch between different musical styles (Miranda and Soucaret, 2008), and later used subject visual gaze direction to allow visual evoked potentials of EEG to control various musical parameters (Miranda et al., 2011). More recently, Miranda et al. (2011) used a statistical analysis of subjective emotions and EEG in an attempt to create an emotion sensor to subconsciously allow users to select music which they associate with more subjectively positive emotions (Eaton et al., 2014). Similarly, Makeig et al. (2011) used EEG and non-EEG signal (scalp muscle and eye movement) from one subject to drive the use of subjective emotions to control a series of musical intervals.

Pham et al. (2005) used slow cortical potentials of EEG to drive control of either ascending or descending pre-set pitch sequences; they used both auditory feedback and visual feedback. While they used tone sequences for feedback, the feedback did not represent a musical context. Using this protocol, they observed significantly better results for visual than auditory feedback. Hinterberger and Baier (2005) used six different frequency bands of EEG signal to drive multiple sound parameters in 10 subjects, some of whom were able to control some sound patterns significantly when prompted with a visual stimulus.

Some of the devices described above which use conscious control can be considered Brain Computer Interfaces (BCIs; Wolpaw and Wolpaw, 2012). BCI research has progressed significantly in advancing towards the goal of using non-invasive EEG scalp electrodes to generate a direct interface from brain signal to a computer to control such actions as moving a cursor on a screen or a word speller (Sellers et al., 2014), driven by signals such as alpha frequency event-related desynchronizations and synchronizations (Roberts et al., 1999; Pfurtscheller et al., 2000). Here, we sought to use the well-described methods of using PDR and motor imagery EEG real-time control to create a new scalar musical instrument, and to measure its accuracy for novices.

In this article, we describe the creation of the Encephalophone, a musical instrument and biofeedback device that uses visual cortex PDR or motor cortex mu rhythm $\mathrm{mu}$ ) to consciously and volitionally control the generation of scalar music. PDR was used due to the simplicity of instructions for novices, as instructions involve opening and closing the eyes. $\mathrm{Mu}$ rhythm was used to generate an instrument that can be controlled without movement (Yuan and He, 2014) for potential applications with patients with motor disabilities. Alpha frequency control using both PDR and mu rhythms has been well described in the BCI literature (Roberts et al., 1999; Pfurtscheller et al., 2000) for non-musical control. We additionally describe experiments demonstrating accuracy for novice users which is significantly higher than random in controlling the instrument by conscious cognitive processes, and show that PDR control was significantly more accurate than mu control.

\section{MATERIALS AND METHODS}

\section{Ethics Statement}

The written informed consent was obtained from each subject prior to testing, and subjects had the opportunity to withdraw from the study at any time. IRB approval (application \#49770) as obtained from the Human Subjects Division of the University of Washington (Seattle, WA, USA).

\section{Recruitment of Subjects}

The subjects were recruited from email and fliers to undergraduate and graduate students at a university setting. Inclusion criterion was healthy adults, and exclusion criteria was age less than 25 or greater than 65 .

\section{EEG Signal Collection}

Figure 1 illustrates the experimental setup (Figure 1). A Mitsar 201 EEG (Mitsar Co., Ltd., St. Petersburg, Russia; distributed by Nova Tech, Inc., Mesa, AZ, USA) and 19-channel ElectroCap electrode cap (Electro-Cap International Inc., Eaton, OH, USA) were used to collect EEG signal utilizing the International 10-20 system of electrode placement (American Electroencephalographic Society, 1994) from 15 healthy human volunteer subjects.

Subjects were positioned in a relaxed, reclining position with a headrest to minimize muscle artifacts, and were positioned facing away from computer screens and other equipment to eliminate any potential for visual feedback. EEG signal at a sampling rate of $500 \mathrm{~Hz}$ was initially processed in a HP Pavilion PC (Hewlett-Packard, Palo Alto, CA, USA) with Mitsar EEG Acquisition software, where filters were applied $(100 \mathrm{~Hz}$ low-pass, $0.5 \mathrm{~Hz}$ high-pass, and $60 \mathrm{~Hz}$ notch filters). Raw EEG signal was visually verified by a physician clinical neurophysiologist for good signal quality and lack of artifacts. EEG data was then streamed in real time to Matlab 


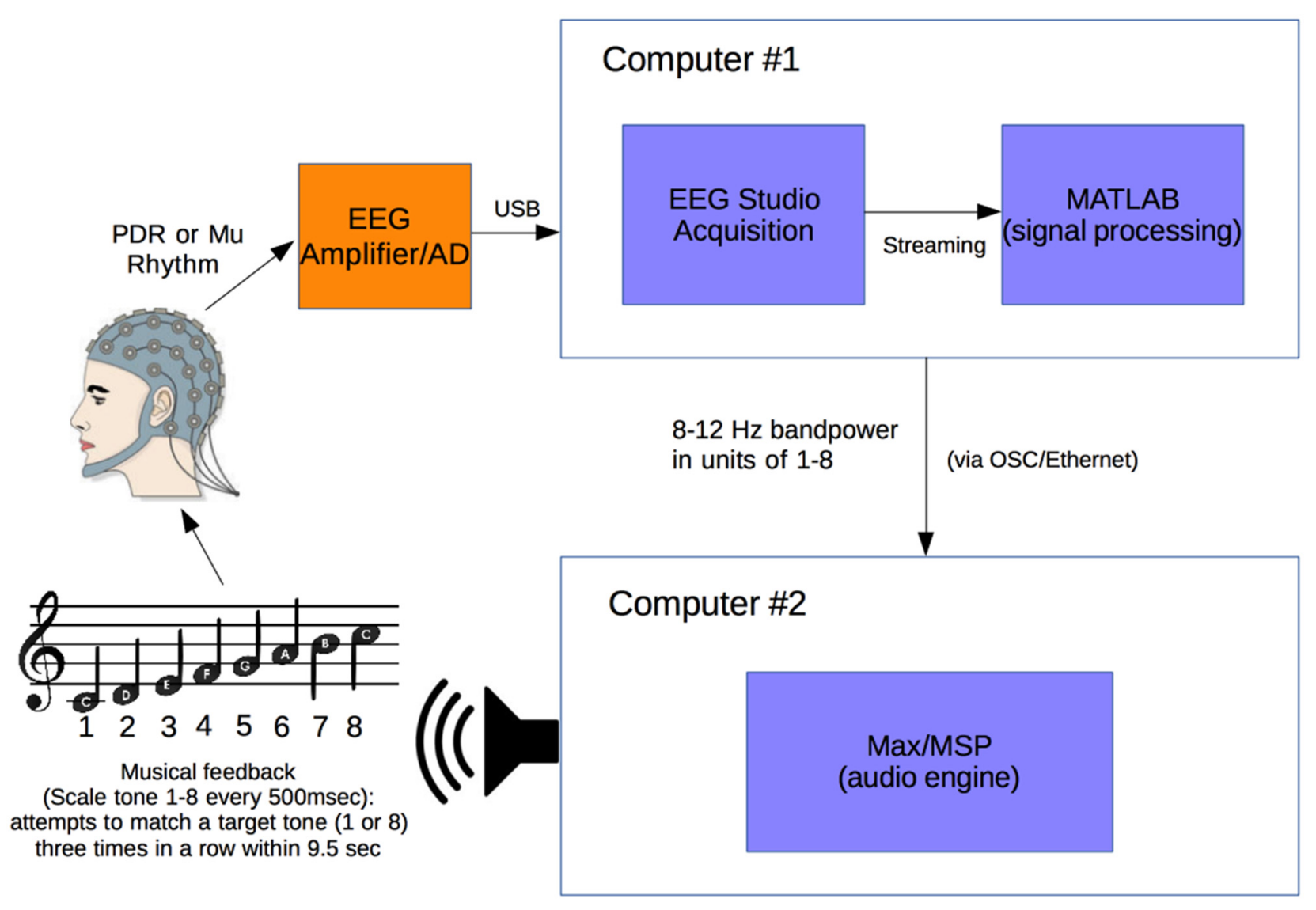

FIGURE 1 | Experimental setup. Electroencephalogram (EEG) signal from subject wearing electrode cap is sent from Mitsar 201 EEG amplifier to Computer \#1 where $8-12 \mathrm{~Hz}$ posterior dominant rhythm (PDR) or Mu power is converted to a value from 1 to 8 . This value from 1 to 8 is sent via OSC to Computer \#2 where it is converted to a musical piano tone in the key of $\mathrm{C}$ (seven tones of $\mathrm{C}$ major scale and octave, from $\mathrm{C} 4$ to $\mathrm{C} 5$ ). Subjects generating tones attempt to match them with a presented target tone.

(The MathWorks, Inc., Natick, MA, USA) via the Mitsar Matlab API.

Matlab scripts for real-time signal processing were created to apply a fourth order Butterworth filter at the 8-12 $\mathrm{Hz}$ band to generate an estimate of power for the PDR in visual cortex from occipital electrode O1, or motor cortex mu rhythm from electrode C3 (international 10-20 system) for right hand motor imagery, in real time. The delay in the system from EEG signal acquisition to Matlab processing was approximately $20 \mathrm{~ms}$. The filter was applied to incoming segments of $500 \mathrm{~ms}$ of data. The bandpass filtered data was rectified and then averaged over the entire segment length to produce a single power estimate for every segment.

\section{Calibration Period}

A calibration was created for each individual subject and each individual trial session of the Encephalophone. The 5 min long calibration period consisted of $2015 \mathrm{~s}$ long alternating cued states ("on" or "off”). For visual cortex PDR, an auditory cue of "on" cued the eyes closed, awake state, and "off" cued the eyes open, awake state. For motor cortex mu rhythm, an auditory cue of "on" cued the awake, resting state and "off" cued the motor imagery (but not actual movement) state: subjects were instructed to imagine right hand grasping and opening at a rate of approximately $1 \mathrm{~Hz}$ as per prior motor imagery BCI methods of Neuper et al. (2006). This calibration period established the range of values of $8-12 \mathrm{~Hz}$ power for an individual subject and individual trial session in the different cued states, then divided these values into eight equal sized "bins", or ranges of values, based on the calibration period alpha power histogram. After calibration, these eight possible values generate the 8 scale degrees of the $\mathrm{C}$ major musical scale including the octave (C4 to C5).

After the calibration period is used to calibrate the instrument to each individual, the device enters the free-running period, during which a value from 1 to 8 is generated every $500 \mathrm{~ms}$ in real-time from the desired $8-12 \mathrm{~Hz}$ frequency power (PDR or $\mathrm{mu}$ rhythm) of the user. Subjects were allowed brief (3 $\mathrm{min}$ ) free-running practice with note generation before accuracy experiments.

This free-running stream of values from 1 to 8 in Matlab is sent at a rate of one value per $500 \mathrm{~ms}$ (120 bpm musical tempo for quarter notes) using Open Sound Control (OSC) 
along an Ethernet cable via a router to a second computer-an Apple MacBook Pro (Apple, Inc., Austin, TX, USA) - where it is received by Max/MSP music generation software (Cycling '74, Walnut, CA, USA). The streaming values from 1 to 8 are used to generate the 8 scale degree notes in the $\mathrm{C}$ major musical scale with a synthesized piano tone (eight notes from C4 to C5).

\section{Accuracy Experiments}

For note accuracy experiments, the subject is presented with a target note of either a high C (C5) or low C (C4). The subject generates one note every $500 \mathrm{~ms}$ and attempts to match the note (C4 or C5) or its nearest neighbor (D4 or B4) three times consecutively. If the note is successfully matched three times consecutively, a "hit" is scored and a reward chord ( $\mathrm{C}$ major) is played, then a new target note is presented. If the subject does not hit the target note three times consecutively within $9.5 \mathrm{~s}$ (19 notes), a "miss" is scored and an error chord (tritone) is played, then a new target note is presented. This results in a chance probability of $19.03 \%$ to score a "hit" over the interval. A total of $300 \mathrm{~s}$, or $5 \mathrm{~min}$, is given for each trial, and the results recorded.

\section{Statistical Analysis}

Statistical analysis was done in conjunction with consultation from the Department of Biostatistics, University of Washington, Seattle, WA, USA. Statistical analysis of $p$-values was performed using the binomial cumulative distribution for individual subjects. The significance of the difference between two means was calculated using the Generalized Linear Mixed Model (GLMM; Breslow and Clayton, 1993) for comparing the PDR condition to the $\mathrm{Mu}$ condition. We used the GLLM test because unlike other non-parametric tests (such as the Wilcoxon signed-rank test), the GLLM tests binary data, clustered data and tests the odds of a hit being significantly different from a miss (rather than the odds that the distributions are significantly different with the Wilcoxon signed-rank test). Statistical analysis of $p$-values for skewness was done using the student's $t$-test with sample population standard deviation.

\section{RESULTS}

Fifteen healthy adult volunteer subjects were trained and tested for musical accuracy using the Encephalophone using both PDR control and motor mu rhythm control (basic subject demographics shown in Table 1). Subjects underwent a $5 \mathrm{~min}$ calibration period, followed by a brief $(3 \mathrm{~min})$ free-run practice period, then a $5 \mathrm{~min}$ accuracy trial for each of PDR and mu control. Results from these musical accuracy experiments were recorded for individual number of hits, trials and percent accuracy for each 5 min trial using PDR control and mu control (summary shown in Table 2).

Subjects using PDR control had an average of 27.4 hits (standard deviation $=11.9$, standard error \pm 3.2 ) in an average of
TABLE 1 | Subject demographics.

\begin{tabular}{lccc}
\hline Subject \# & Age & Gender & $\begin{array}{c}\text { Years musical } \\
\text { training }\end{array}$ \\
\hline 1 & 27 & $\mathrm{M}$ & 18 \\
2 & 63 & $\mathrm{M}$ & 3 \\
3 & 44 & $\mathrm{M}$ & 18 \\
4 & 28 & $\mathrm{~F}$ & 18 \\
5 & 28 & $\mathrm{~F}$ & 7 \\
6 & 42 & $\mathrm{~F}$ & 14 \\
7 & $\mathrm{M}$ & 0 \\
8 & 37 & $\mathrm{M}$ & 0 \\
9 & 35 & $\mathrm{~F}$ & 5 \\
10 & 38 & $\mathrm{M}$ & 25 \\
11 & 31 & $\mathrm{M}$ & 19 \\
12 & 27 & $\mathrm{~F}$ & 11 \\
13 & 25 & $\mathrm{M}$ & 8 \\
14 & 27 & $\mathrm{M}$ & 6 \\
15 & 48 & $\mathrm{~F}$ & 10 \\
Average & 32 & & 10.80 \\
\hline
\end{tabular}

38.7 trials, resulting in an average of 67.1\% accuracy (Figure 2A, standard deviation $=17.42 \%$, standard error $\pm 4.5 \%)$. Subjects using mu control had an average of 20.6 hits (standard deviation $=5.7$, standard error \pm 1.5 ) in an average of 35.6 trials, resulting in an average of $57.1 \%$ accuracy (Figure 2B, standard deviation $=11.2 \%$, standard error $\pm 3.0 \%)$. Each individual subject scored significantly higher than random in accuracy for both PDR and mu control (Figure 2): $p$ values ranged from $6.3 \times 10^{-36}$ to $2.8 \times 10^{-3}$. Additionally, PDR accuracies (average $67.1 \%)$ were significantly higher $\left(p=1.4 \times 10^{-4}\right)$ than $\mathrm{Mu}$ accuracies (average 57.1\%).

In order to assess for individual subject bias skewed towards particular notes, histograms of note generation during each 5 min testing session were created, and skewness calculated (Figure 3). If an individual exclusively generated a high or low note, for example, this bias would result in scoring a hit in 50\% of note trials. Extreme skew bias could occur, for example, if no alpha frequency signal was recorded during calibration. Note that the task itself of hitting one of two notes at the extremes of the note range may generate skewness towards the extremes (notes 1 and 8). There was a range of skew bias, with the most significant skew bias towards low note generation (skewness +2.42 , $p=2.1 \times 10^{-9}$ ) with subject \#13/PDR, and toward high note generation (skewness $-0.57, p=6.8 \times 10^{-3}$ ) with subject \#10/PDR. In these two most biased cases, if we look at their accuracy with the non-biased target note alone (i.e., throw out biased target note trials), they score $61.1 \%$ $\left(p=1.10 \times 10^{-6}\right)$, and $67 \%\left(p=1.80 \times 10^{-8}\right)$ note matching accuracy, respectively, significantly above random chance (19.03\%).

We also looked at the correlation between PDR hits and accuracy, and mu hits and accuracy, with years of musical training (Figure 4). There was a moderate positive relationship between increased PDR hits and accuracy (correlation values 0.58 and 0.41 , respectively) - but not mu hits and accuracy (correlation values -0.16 and -0.11 , respectively)-with increasing years of musical training. 
TABLE 2 | Individual subject results from accuracy experiments.

\begin{tabular}{|c|c|c|c|c|c|c|c|c|}
\hline Subject \# & PDR hits & PDR trials & PDR \% & $p$ value & Mu hits & Mu trials & Mu \% & $p$ value \\
\hline 1 & 16 & 35 & 45.7 & 7.41E-05 & 17 & 35 & 48.6 & 1.69E-05 \\
\hline 2 & 23 & 36 & 63.9 & 5.69E-10 & 32 & 41 & 78.0 & $3.11 \mathrm{E}-17$ \\
\hline 3 & 33 & 39 & 84.6 & 6.57E-20 & 25 & 39 & 64.1 & $1.08 \mathrm{E}-10$ \\
\hline 4 & 23 & 40 & 57.5 & $1.28 \mathrm{E}-08$ & 27 & 39 & 69.2 & $1.20 \mathrm{E}-12$ \\
\hline 5 & 30 & 41 & 73.2 & $8.22 \mathrm{E}-15$ & 19 & 35 & 54.3 & 6.36E-07 \\
\hline 6 & 52 & 55 & 94.5 & 6.34E-36 & 28 & 38 & 73.7 & $3.47 \mathrm{E}-14$ \\
\hline 7 & 30 & 38 & 78.9 & $1.40 \mathrm{E}-16$ & 16 & 33 & 48.5 & 2.81E-05 \\
\hline 8 & 14 & 32 & 43.8 & $3.20 \mathrm{E}-04$ & 17 & 33 & 51.5 & $5.72 \mathrm{E}-06$ \\
\hline 9 & 12 & 31 & 38.7 & 2.79E-03 & 27 & 40 & 67.5 & $3.28 \mathrm{E}-12$ \\
\hline 10 & 52 & 55 & 94.5 & 6.34E-36 & 19 & 34 & 55.9 & 3.32E-07 \\
\hline 11 & 34 & 41 & 82.9 & 7.95E-20 & 19 & 33 & 57.5 & 1.67E-07 \\
\hline 12 & 19 & 33 & 57.6 & 1.67E-07 & 15 & 33 & 45.5 & $1.24 \mathrm{E}-04$ \\
\hline 13 & 24 & 27 & 88.9 & $1.71 \mathrm{E}-11$ & 12 & 31 & 38.7 & 2.79E-03 \\
\hline 14 & 25 & 41 & 61.0 & $5.65 \mathrm{E}-10$ & 17 & 35 & 48.6 & 1.69E-05 \\
\hline 15 & 23 & 37 & 62.2 & 1.32E-09 & 19 & 35 & 54.3 & 6.36E-07 \\
\hline Average & 27.4 & 38.7 & 67.1 & & 20.6 & 35.6 & 57.1 & \\
\hline Random & & & 19.03 & & & & 19.03 & \\
\hline
\end{tabular}

Number of hits, number of trials, and percent (\%) accuracy with p values for both PDR control and mu control.

\section{DISCUSSION}

This article describes the creation of the Encephalophone, a musical instrument and biofeedback device, which uses either PDR or mu rhythm EEG signal to control notes of a musical scale in real time. We describe testing 15 normal subjects novice to the device in experiments to test accuracy in hitting a target note, and our results show each subject scoring significantly higher than random, with the average score much higher than random for both PDR and mu control. $\mathrm{Mu}$ control average accuracy is comparable with that previously shown $(56 \%)$ by studies using motor imagery mu control (Neuper et al., 2005). PDR control showed significantly higher accuracy than mu control for these novices, as might be expected given the more straightforward task of opening and closing eyes-as opposed to increasing and decreasing motor imagery. We additionally looked at skew bias for individual notes for each subject, and found most subjects without large skew bias. Even those subjects with larger biases were able to score hits at both ends of the note range. We additionally found a moderate positive correlation between years of musical training and PDR accuracy, but not mu accuracy.

These studies demonstrate that the Encephalophone allows novices to have some cognitive volitional control of generation of musical notes in real time, without movement. We believe these results and the creation of this device is of significant interest for several reasons. First, the Encephalophone represents a novel musical instrument that uses EEG control to create scalar music in real-time, and allows some basic accuracy that has been experimentally tested here. Second, given the known potential for significant improvement with training in mu-based BCI devices (Neuper et al., 2006), novices such as those tested here have the potential with continued training to significantly improve accuracy and facility with the instrument. The use of scalar musical tones-rather than non-musical sound or visual biofeedback-may confer a training advantage: the benefits of music for arousal motivation for both training and therapeutics have been shown (Bergstrom et al., 2014). Third, the use of a musical feedback-based EEG device with
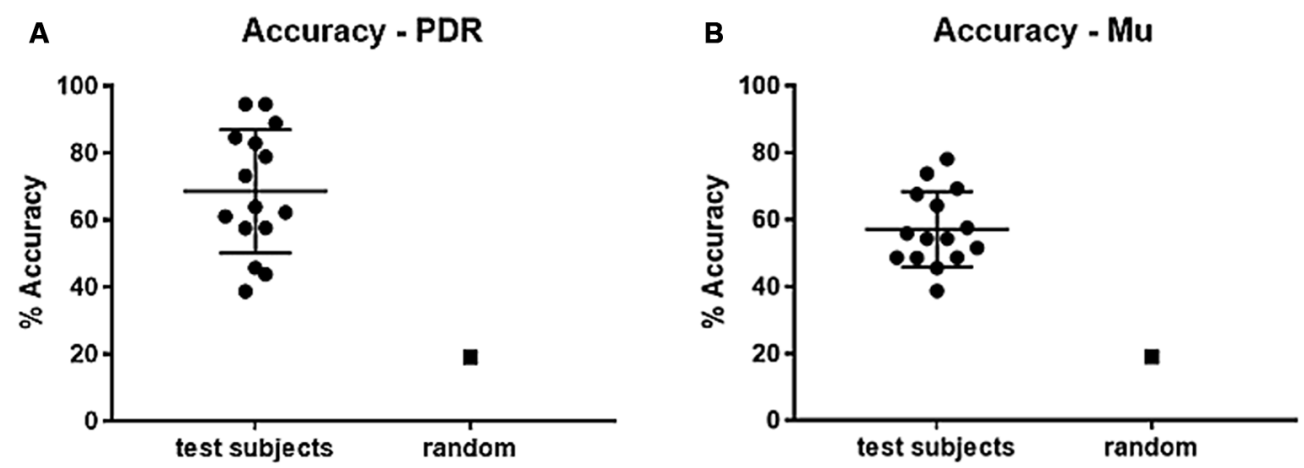

FIGURE 2 | Percent accuracy for PDR and Mu control. Individual subjects were given 5 min to hit as many target notes as possible using either PDR control or Mu control. Scatter plots of results of all subjects were generated (bars represent mean and standard deviation), with random (chance) control, for each of: (A) Percent accuracy using PDR control (standard error \pm 4.7 ). (B) Percent accuracy using mu control (standard error \pm 3.0 ). 
A
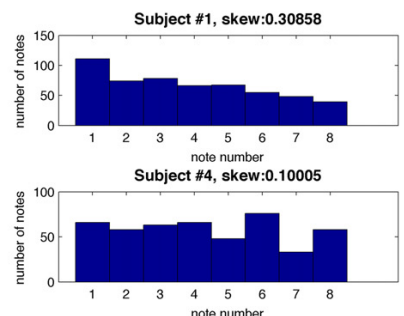

Subject $\# 7$, skew: 0.0468
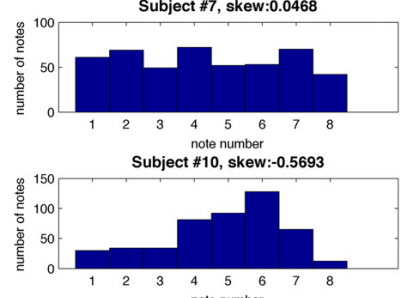

Subject \#13, skew:2.418

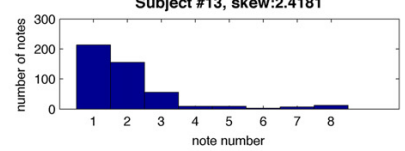

B

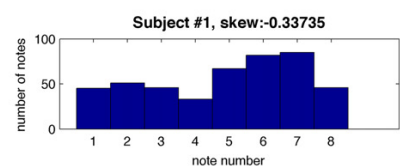

Subject \#4, skew:-0.0026239

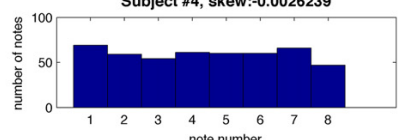

Subject \#7, skew:0.29961

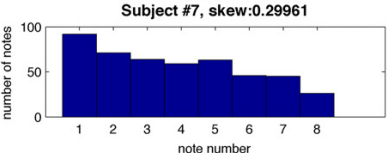

Subject \#10, skew:0.046251

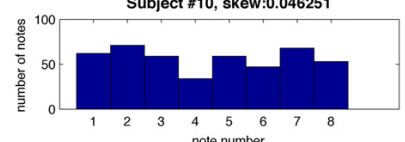

Subject \#13, skew:-0.16589

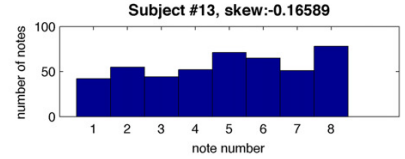

Skew Bias - PDR

Subject \#2, skew:-0.066529

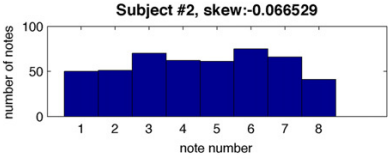

Subject \#5, skew:0.036771

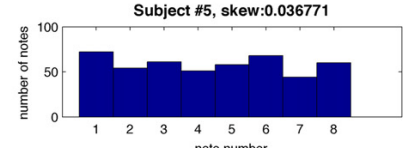

Subject \#8, skew:0.14591

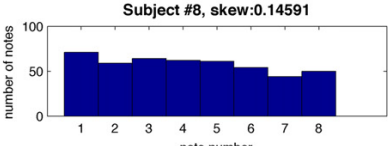

Subject \#11, skew:-0.24645

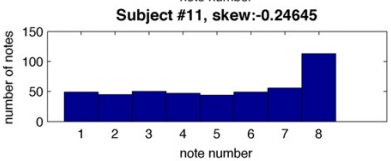

Subject \#14, skew:-0.48573

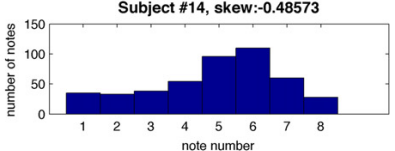

Skew Bias - Mu

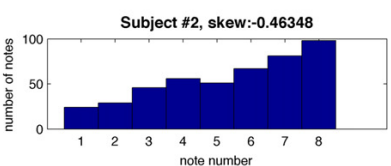

Subject \#5, skew:0.046322

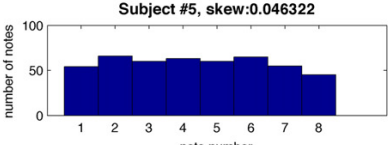

Subject \#8, skew:-0.50266

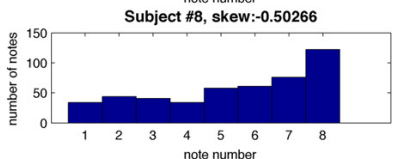

Subject \#11, skew:-0.41023

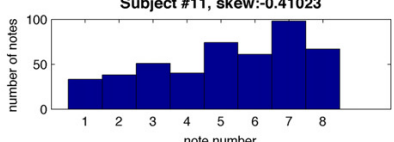

note number
Subject \#14, skew:0.29581

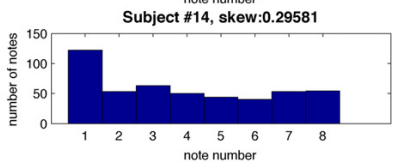

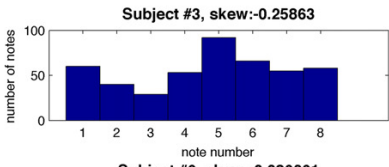

Subject \#6, skew:-0.086261

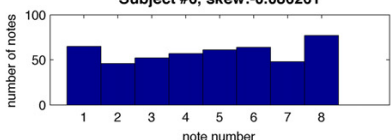

Subject \#9, skew:0.091915

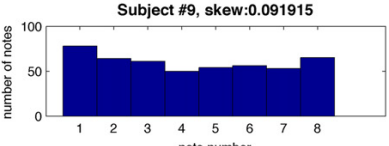

Subject \#12, skew:-0.28666

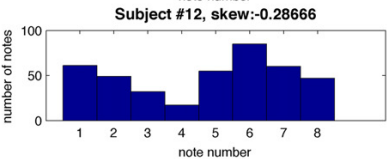

Subject \#15, skew:0.21681

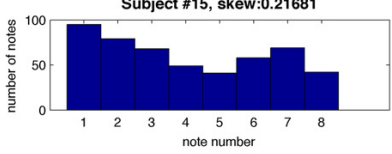

note number

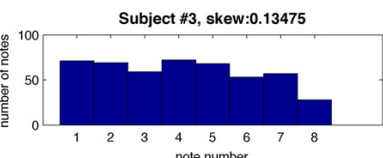

Subject \#6, skew:0.0093401

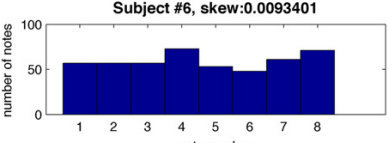

Subject \#9, skew:0.01912

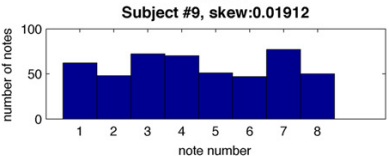

Subject \#12, skew:0.018907

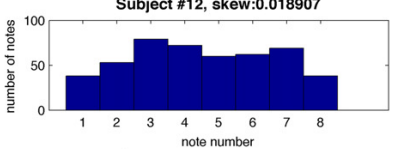

note number
Subject \#15, skew:-0.50918

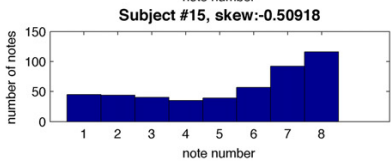

FIGURE 3 | Skew bias for individual notes for each subject. Histograms for each individual subject showing frequency ( $y$ axis) of each of eight possible musical notes ( $x$ axis) as well as skewness values during testing for: (A) PDR control experiment and (B) Mu control experiment.

responsiveness noticeable to the user may hold promise for patients-such as those with locked-in syndrome-who are severely incapacitated and may be more likely to respond to auditory (and specifically musical) stimulus and feedback than to visual stimulus and feedback. This is particularly so for those who may have visual impairment (e.g., cortical blindness), and particularly to those who played music before their injury.

Previously, others have reported use of BCI to control not only visual output (e.g., cursor on a computer screen) but also sound, and reported better control and accuracy with visual rather than (non-musical) auditory feedback 

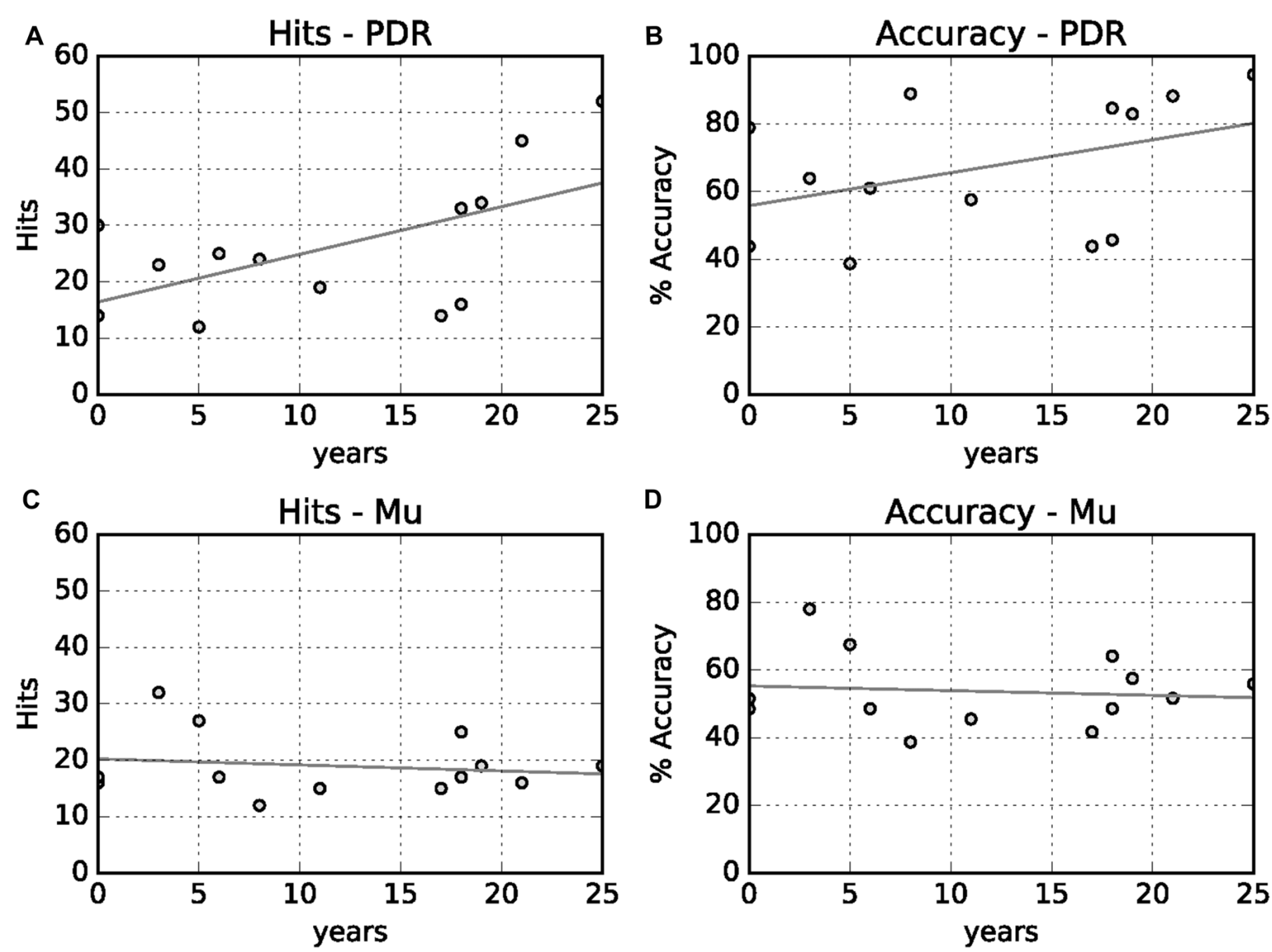

FIGURE 4 | Relationship between years of musical training with hits and accuracy. (A) Years musical training vs. PDR hits (correlation value $0.58, p=0.01$ ). (B) Years musical training vs. PDR accuracy (correlation value $0.41, p=0.06$ ). (C) Years musical training vs. mu hits (correlation value $0.16, p=0.28$ ). (D) Years musical training vs. mu accuracy (correlation value $-0.11, p=0.35$ ).

(Nijboer et al., 2008). However, Bergstrom et al. (2014) showed musical biofeedback to be better than either simple passive music listening or non-musical sonification biofeedback for control of physiological arousal state. Here we report reasonable control with virtually no training, using scalar musical tone feedback rather than non-musical auditory feedback. Thus we hope that with further training involving musical accompaniment between testing sessions, the musical context provided will greatly improve learning and accuracy of control. This will be tested in future experiments with serial training, as well as testing with more note target options ( 3 or 4 possible notes to match rather than 2).

This device is being used as a novel improvisational musical instrument in live performance, accompanied by small ensembles of musicians. Future development will include using multiple soloists performing with Encephalophones together, in a call and response improvisation, as well as performers improvising not only with musical scales, but also with timbre or chordal improvisation. Furthermore, work in computer music using conscious control of sound spatialization is being explored.

Diagnostically, the Encephalophone might prove useful in auditory monitoring of clinical EEG applications, such as auditory seizure detection, as described by Loui et al. (2014). Therapeutically, we also plan on using the Encephalophone in trials of cognitive rehabilitation and neurologic music therapy with patients with motor disabilities (but with at least one intact motor cortex). It has already been demonstrated that neurologic music therapy improves executive function in traumatic brain injury rehabilitation (Thaut et al., 2009). Other patients who might thus benefit would include patients suffering from amyotrophic lateral sclerosis (ALS), brainstem stroke, or traumatic amputation (such as war veterans). The ability to generate music using a portion of the brain that is no longer able to control motor movement of limbs may be beneficial for emotional and cognitive rehabilitation. Also, combining the Encephalophone with physical therapy may improve motor rehabilitation, and cortical "rewiring" of motor circuits may 
allow new motor output pathways for regaining some motor control.

\section{AUTHOR CONTRIBUTIONS}

TAD contributed the largest amount to all aspects of the work-conception of design of the Encephalophone, experimental design, acquisition of the data, analysis of the data, as well as drafting and editing the manuscript. JP contributed to all of this aspects of the work, but particularly experimental design, analysis of data and drafting and editing the manuscript.

\section{REFERENCES}

Adrian, E. D., and Matthews, B. H. (1934). The interpretation of potential waves in the cortex. J. Physiol. 81, 440-471. doi: 10.1113/jphysiol.1934.sp003147

American Electroencephalographic Society. (1994). Guideline thirteen: guidelines for standard electrode position nomenclature. J. Clin. Neurophysiol. 11, 111-113. doi: 10.1097/00004691-199401000-00014

Berger, H. (1929). Über das elektrenkephalogramm des menschen (On the human electroencephalogram). Archiv. Psychiatr. Nervenkr. 87, 527-570. doi: 10.1007/BF01797193

Bergstrom, I., Seinfeld, S., Arroyo-Palacios, J., Slater, M., and Sanchez-Vives, M. V. (2014). Using music as a signal for biofeedback. Int. J. Psychophysiol. 93, 140-149. doi: 10.1016/j.ijpsycho.2013.04.013

Breslow, N., and Clayton, D. (1993). Appoximate inference in generalized linear mixed models. J. Am. Stat. Assoc. 88, 9-25. doi: 10.1080/01621459.1993. 10594284

Brouse, A., Filatriau, J., Gaitanis, K., Lehembre, R., Macq, B., Miranda, E. R., et al. (2006). Use of spectral analysis of multiple frequency bands of EEG brain waves to generate sound. An instrument of sound and visual creation driven by biological signals. eNTERFACE summer workshop. Available online at: http://www.enterface.net

Eaton, J., Williams, D., and Miranda, E. (2014). "Affective jukebox: a confirmatory study of EEG emotional correlates in response to musical stimuli," in Proceedings of Joint ICMC. 11th Sound and Music Conference, 14th International Computer Music Conference (Greece: University of Athens).

Hinterberger, T., and Baier, G. (2005). Parametric orchestral sonification of EEG in real time. IEEE Multimed. 12, 70-79. doi: 10.1109/mmul.2005.36

Loui, P., Koplin-Green, M., Frick, M., and Massone, M. (2014). Rapidly learned identification of epileptic seizures from sonified EEG. Front. Hum. Neurosci. 8:820. doi: $10.3389 /$ fnhum.2014.00820

Lucier, A. (1965). Music for solo performer. Available online at: https://www.youtube.com/watch?v=bIPU2ynqy2Y

Makeig, S., Leslie, G., Mullen, T., Sarma, D., Bigdely-Shamlo, N., and Kothe, C. (2011). "First demonstration of a musical emotion BCI," in Proceedings 4th International Conference, ACII 2011, Affective Computing and Intelligent Interaction - Volume Part II (Berlin, Heidelberg: Springer-Verlag), 487-496.

Miranda, E. R., Magee, W. L., Wilson, J. J., Eaton, J., and Palaniappan, R. (2011). Brain-computer music interfacing (BCMI): from basic research to the real world of special needs. Music Med. 3, 134-140. doi: 10.1177/19438621113 99290

Miranda, E. R., and Soucaret, V. (2008). "Mix-it-yourself with a braincomputer music interface 2008," Proceedings of 7 th International Conference on Disability, Virtual Reality and Associated Technologies (ICDVRAT) and ArtAbilitation (Porto, Portugal).

Neuper, C., Müller-Putz, G. R., Scherer, R., and Pfurtscheller, G. (2006). Motor imagery and EEG-based control of spelling devices and neuroprostheses. Prog. Brain Res. 159, 393-409. doi: 10.1016/s0079-6123(06)59025-9
JS contributed to all of this aspects of the work, particularly in experimental stimulus design, acquisition of data, analysis of data and drafting and editing the manuscript. FD contributed the second largest amount to all aspects of the work, particularly in design of the Encephalophone, experimental design, analysis of the data, as well as drafting and editing the manuscript.

\section{FUNDING}

This research received no outside funding. The authors received no specific funding for this work.

Neuper, C., Scherer, R., Reiner, M., and Pfurtscheller, G. (2005). Imagery of motor actions: differential effects of kinesthetic and visual-motor mode of imagery in single-trial EEG. Cogn. Brain Res. 25, 668-677. doi: 10.1016/j.cogbrainres.2005. 08.014

Nijboer, F., Furdea, A., Gunst, I., Mellinger, J., McFarland, D. J., Birbaumer, N., et al. (2008). An auditory brain-computer interface (BCI). J. Neurosci. Methods 167, 43-50. doi: 10.1016/j.jneumeth.2007.02.009

Pfurtscheller, G., Neuper, C., Guger, C., Harkam, W., Ramoser, H., Schlögl, A., et al. (2000). Current trends in graz brain-computer interface (BCI) research. IEEE Trans. Rehabil. Eng. 8, 216-219. doi: 10.1109/86. 847821

Pham, M., Hinterberger, T., Neumann, N., Kübler, A., Hofmayer, N., Grether, A., et al. (2005). An auditory brain-computer interface based on the self-regulation of slow cortical potentials. Neurorehabil. Neural Repair 19, 206-218. doi: $10.1177 / 1545968305277628$

Roberts, S. J., Penny, W., and Rezek, I. (1999). Temporal and spatial complexity measures for electroencephalogram based brain-computer interfacing. Med. Biol. Eng. Comput. 37, 93-98. doi: 10.1007/bf025 13272

Rosenboom, D. (1975). "Statement on: music for solo performer," in Biofeedback and the Arts: Results of Early Experiments, ed. D. Rosenboom (Vancouver, BC: Aesthetic Research Centre of Canada), 60-61.

Sellers, E. W., Rayn, D. B., and Hauser, C. K. (2014). Noninvasive brain-computer interface enables communication after brainstem stroke. Sci. Transl. Med. 6:257re7. doi: 10.1126/scitranslmed.3007801

Thaut, M. H., Gardiner, J. C., Holmberg, D., Horwitz, J., Kent, L., Andrews, G., et al. (2009). Neurologic music therapy improves executive function and emotional adjustment in traumatic brain injury rehabilitation. Ann. N Y Acad. Sci. 1169, 406-416. doi: 10.1111/j.1749-6632.2009. 04585.x

Wolpaw, J. R., and Wolpaw, E. W. (2012). Brain-Computer Interfaces: Principles and Practice. Oxford, New York, NY: Oxford University Press.

Yuan, H., and He, B. (2014). Brain-computer interfaces using sensorimotor rhythms: current state and future perspectives. IEEE Trans. Biomed. Eng. 61, 1425-1435. doi: 10.1109/TBME.2014.2312397

Conflict of Interest Statement: The authors declare that the research was conducted in the absence of any commercial or financial relationships that could be construed as a potential conflict of interest.

Copyright (c) 2017 Deuel, Pampin, Sundstrom and Darvas. This is an open-access article distributed under the terms of the Creative Commons Attribution License (CC BY). The use, distribution or reproduction in other forums is permitted, provided the original author(s) or licensor are credited and that the original publication in this journal is cited, in accordance with accepted academic practice. No use, distribution or reproduction is permitted which does not comply with these terms. 